\title{
Exploitation et traits de menaces des grenouilles d'un intérêt socio- économique dans la province du Ganzourgou au Burkina Faso, Afrique de l'Ouest
}

\author{
Nessan Désiré COULIBALY ${ }^{1 *}$ et Boudoulaye ZIGUI²

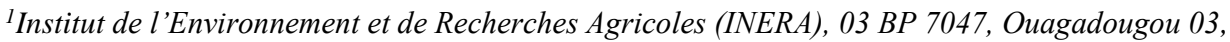 \\ Burkina Faso. \\ ${ }^{2}$ Ecole Nationale d'Elevage et de la Santé Animale (ENESA), 03 BP 7026, Ouagadougou 03, Burkina Faso. \\ *Auteur correspondant ; E- mail : nessandesirec@ gmail.com; Tél. (+226) 70739899
}

\begin{tabular}{ccc}
\hline Received: 05-11-2020 & Accepted: 28-05-2021 & Published: 30-06-2021 \\
\hline
\end{tabular}

\section{RESUME}

Les grenouilles font partie des ressources halieutiques dont l'exploitation n'est ni règlementée ni encadrée. Ce vide technique et juridique expose ces grenouilles à une exploitation anarchique pouvant conduire à leur extinction. Notre étude avait pour but d'identifier les grenouilles d'intérêt socio-économique, leur mode d'exploitation et les facteurs de menace dans la province du Ganzourgou. Des enquêtes ont été menées auprès des acteurs exploitant cette ressource. Quelques sites d'exploitation et de commercialisation des produits ont aussi été visités. Deux espèces de grenouilles sont rencontrées et exploitées artisanalement en contre-saison. Les grenouilles récoltées sont valorisées dans l'alimentation humaine, en pharmacopée et dans les rites culturels. Les facteurs de menaces identifiés et qui pèsent sur cette population sauvage de grenouilles est l'utilisation des substances agrochimiques et chimiques en production agricole et minière. D'autres facteurs tels que la pénurie d'eau, la modification ou la perte de leurs habitats sont cités. Toutefois, la menace la plus importante décelée serait le vide juridique qui ne permet pas de réguler ni d'encadrer l'exploitation de cette ressource naturelle. Les grenouilles sont sous la menace des facteurs climato-anthropiques, d'où la nécessité d'entrevoir dès à présent les stratégies de leur conservation, voire de leur domestication en vue d'une production durable et maitrisée.

(C) 2021 International Formulae Group. All rights reserved.

Mots clés : Ressources aquatiques, valorisation, dégradation, Ganzourgou, Burkina Faso.

\section{Exploitation and threat traits of frogs of socio-economic interest in the province of Ganzourgou in Burkina Faso, West Africa}

\begin{abstract}
Frogs are part of fishery resources that are either regulated or controlled. This technical and regulatory void exposed these frogs to an anarchic exploitation which may lead to their extinction. Our study aimed to identify frogs of socio-economic interest, their mode of exploitation and the threat factors in the province of Ganzourgou. Surveys were carried out among frog stakeholders. Some sites for operating and marketing products were also visited. Two species of frogs are encountered and traditionally exploited in the off-season. The harvest frogs are valued in human food, in pharmacopoeia and in cultural rites. The identified threat factors
\end{abstract}


that hangs on this wild population of frogs is the significant use of agrochemicals and chemicals in agricultural and mining production. Others factors such as water scarcity and the modification or loss of their habitats are cited. However, the most important threat detected would be the legal vacuum which does not allow to regulated or supervise the exploitation of this natural resource. Frogs are under threat from climatic and anthropogenic factors, hence the need to foresee now the strategies of their conservation or even their domestication with a view to a sustainable and controlled production.

(C) 2021 International Formulae Group. All rights reserved.

Keywords: Aquatic resources, valuation, degradation, Ganzourgou, Burkina Faso.

\section{INTRODUCTION}

Les grenouilles revêtent une importance dans le monde sur le plan alimentaire (Teixeira et al., 2001 ; Altherr et al., 2011), écologique (Mohneke et al., 2010b ; Penner et al., 2010), économique (Oungbe et al., 2018) et culturel (Onadeko et al., 2011 ; Efenakpo et al., 2016). Plus récemment, Altherr et al. (2011) ont rapporté le développement d'un marché florissant en relation avec l'utilisation des grenouilles comme nouveaux animaux de compagnie (NAC). En Afrique, la place des grenouilles est connue dans l'alimentation des populations locales (Mohneke et al., 2010a, 2010c ; Altherr et al., 2011 ; Efenakpo et al., 2016 ; Kia et al., 2018 ; Oungbe et al., 2018) ainsi que dans les utilisations en pharmacopée ou la médecine traditionnelle (Mohneke et al., 2010 a, 2010b ; Altherr et al., 2011). Elles sont également utilisées dans les aspects culturels (Mohneke et al., 2010b ; Onadeko et al., 2011 ; Efenakpo et al., 2016 ; MRAH, 2018).

Au Burkina Faso, pays à climat aride, les grenouilles sont rencontrées dans tous les écosystèmes humides (MRAH, 2018). Ces derniers sont constitués par des cours d'eau saisonniers (Nazinon, Nakanbé, Béli), des cours d'eau pérennes (Mouhoun, Léraba, Comoé, Pendjari) et environ 2100 retenues d'eau de surface (Dipama, 2010). Ces grenouilles ne sont malheureusement pas valorisées dans toutes les régions d'occurrence (MRAH, 2018). Dans la région administrative du Plateau central et plus précisément dans la province du Ganzourgou, les grenouilles font l'objet d'une exploitation artisanale à côté des poissons (Mohneke et al., 2010b; Marina, 2016 ; Zigui, 2020). L'espèce de grenouille la plus rencontrée dans les prises est
Hoplobatrachus occipitalis, Günther 1858, appelée «louanga» par les mossis qui constituent le groupe ethnique majoritaire vivant dans cette province (MINEFID, 2015). La présence d'autres espèces a été rapportée. Il s'agit de Pyxicephalus edulis et de Xenopus muelleri (Mohneke et al., 2010c; WAR et SOMISA, 2019; Zigui, 2020). La consommation des grenouilles à grande échelle constitue l'un des traits culturels typiques aux habitants natifs de cette province.

La province du Ganzourgou a une vocation essentiellement agricole caractérisée par une utilisation importante de produits agrochimiques (engrais, herbicides, pesticides) pour la fertilisation des sols pauvres et la protection des cultures de rente ainsi que des cultures maraîchères (MINEFID, 2015). L'exploitation minière, consommatrice de substances chimiques, connait également un développement significatif ces dernières années dans cette province. Le contexte climatique précaire et l'utilisation de toutes ces substances chimiques semblent constituer des traits de menace pour les stocks de populations sauvages de grenouilles.

Les données relatives à l'exploitation de ces grenouilles sont ténues et fragmentaires lorsqu'elles existent. C'est pour mieux appréhender cette exploitation de grenouilles et les principaux traits de menaces, que la présente étude est entreprise dans la province $\mathrm{du}$ Ganzourgou. Elle vise à identifier la diversité des grenouilles d'un intérêt socioéconomique rencontrées dans la province, à caractériser leur mode d'exploitation et de valorisation et identifier les facteurs de menaces qui pèsent sur cette ressource bioaquatique. 


\section{MATERIEL ET METHODES \\ Présentation de la zone d'étude}

La province du Ganzourgou est située dans la région du Plateau Central au Burkina Faso entre les coordonnées de $12^{\circ} 42$ et $11^{\circ} 32$ de latitude Nord et $0^{\circ} 25$ et $1^{\circ} 08$ de latitude Ouest (Figure 1). Elle couvre une superficie de $4168 \mathrm{~km}^{2}$, soit près de la moitié de la superficie totale de la région du Plateau Central (8605 $\mathrm{km}^{2}$ ). Sa population qui était de 393778 habitants en 2014 est dominée par les femmes (53\%) et les jeunes. Le climat de la province est de type nord-soudanien, marqué par une saison pluvieuse de mai/juin à octobre et une saison sèche beaucoup plus longue allant de novembre à début mai. Les précipitations sont irrégulières, mal réparties dans le temps et dans l'espace avec une hauteur de 650 à $850 \mathrm{~mm} / \mathrm{an}$ pour moins de 60 jours de pluies (MINEFID, 2015). Les températures oscillent entre $13{ }^{\circ} \mathrm{C}$ (décembre/janvier) à $45^{\circ} \mathrm{C}$ au mois de mai. La végétation, de type savane arbustive (sur les plateaux), arborée dans les vallées et de type galerie forestière le long des cours d'eau est la résultante d'une dégradation d'origine climatique (sécheresse) mais surtout anthropique. Le réseau hydrographique est constitué par des bas-fonds et des cours d'eau intermittents, largement tributaires des précipitations. Le tarissement en saison sèche est la caractéristique principale des écosystèmes lotiques de la province à savoir le Nakambé, la Dougoula-moundi, le Bomboré et la Guibga. La province compte 45 retenues d'eau de surface (barrages) qui constituent une potentialité pour le développement des cultures irriguées et une amplification de l'habitat des ressources animales aquatiques comme les amphibiens. L'agriculture et l'élevage constituent les principales activités de production. L'agriculture est de type traditionnel, articulée autour des cultures pluviales (céréales notamment) et des cultures de contre-saison, le maraîchage dans les périmètres aménagés autour des retenues d'eau de surface. Ces cultures maraîchères et celles de rente reçoivent les engrais minéraux (NPK, urée, traitements phytosanitaires) en raison de la pauvreté des sols dans la province. La province dispose également d'un important potentiel minier avec de nombreux sites (26) d'orpaillage artisanal dont deux (2) exploitations industrielles. Le caractère saisonnier des cours et plans d'eau dans la province constitue le principal handicap pour le développement de l'exploitation halieutique et la pratique de l'aquaculture. La retenue d'eau de la commune de Mogtédo (290 ha) qui est la plus importante de la province, manque de données pondérales sur les captures halieutiques (MINEFID, 2015).

\section{Collecte et analyse des données}

L'étude s'est déroulée d'août 2018 à juillet 2020 avec des sorties alternées (saison sèche et saison des pluies) sur les sites de pêche, la visite des principaux marchés (Zorgho et Mogtédo), l'examen des paniers de pêche et des entretiens avec les acteurs de terrain. Cinq catégories d'acteurs ont été identifiées et enquêtées. Il s'agit des services techniques déconcentrés de l'administration (agriculture, élevage, foresterie, santé) et des collectivités territoriales (municipalités), des organisations faîtières de pêcheurs, des femmes transformatrices des produits de pêche, des consommateurs et de personnes ressources (notables, anciens pêcheurs). Les effectifs de toutes ces catégories sont indiqués au Tableau 1.

Cinq (5) questionnaires semi-structurés ont été élaborés et administrés aux personnes listées au Tableau 1. Les questionnaires élaborés à l'endroit des acteurs directs (pêcheurs et transformatrices) ont été administrés à l'occasion d'un focus group. Des interviews/entretiens individuels ont été la forme d'administration du questionnaire aux autres personnes enquêtées (services techniques, consommateurs, personnes ressources). Les questionnaires ont portés sur cinq thématiques dont la diversité des espèces rencontrées dans la province. Pour cette thématique, des planches photographiques de diverses espèces de grenouilles rencontrées au Burkina Faso ont été présentées aux pêcheurs, aux personnes ressources et aux responsables des services techniques pour identifier les espèces de grenouilles rencontrées dans la province et les connaissances endogènes sur la 
valeur des grenouilles. La description des espèces présentées sur ces planches photographiques est extraite des ouvrages de différents auteurs (Mohneke et al., 2010b; Godome et al., 2018 ; Oungbe et al., 2018). Les quatre autres thématiques ont trait à l'écologie (habitats et zone d'occurrence des grenouilles), l'exploitation proprement dite (période et durée de pêche ; techniques de capture y compris les engins et méthodes utilisés), les aspects socioéconomiques et culturels (consommation, commercialisation/prix, valeurs culturelles) et enfin les traits de menaces qui pèsent sur ces grenouilles dans la province. Ce questionnaire a été complété par les visites de marché
(Zorgho et Mogtédo) et de certains sites humides (lac de barrage de Mogtédo, lac de barrage de Zoungou, lacs de barrage de Sanbrado, de Tanlouka, de Koakin et le cours d'eau du Guibga).

Les données collectées à travers les questionnaires, interviews et visites de sites ont été analysées en utilisant les statistiques descriptives. Le tableur/logiciel EXCEL version 2013 a été utilisé pour tracer les histogrammes ou les graphiques en camemberts afin d'analyser les caractéristiques des habitats et des engins de capture des grenouilles dans la province ou les sources de déclins de la population.

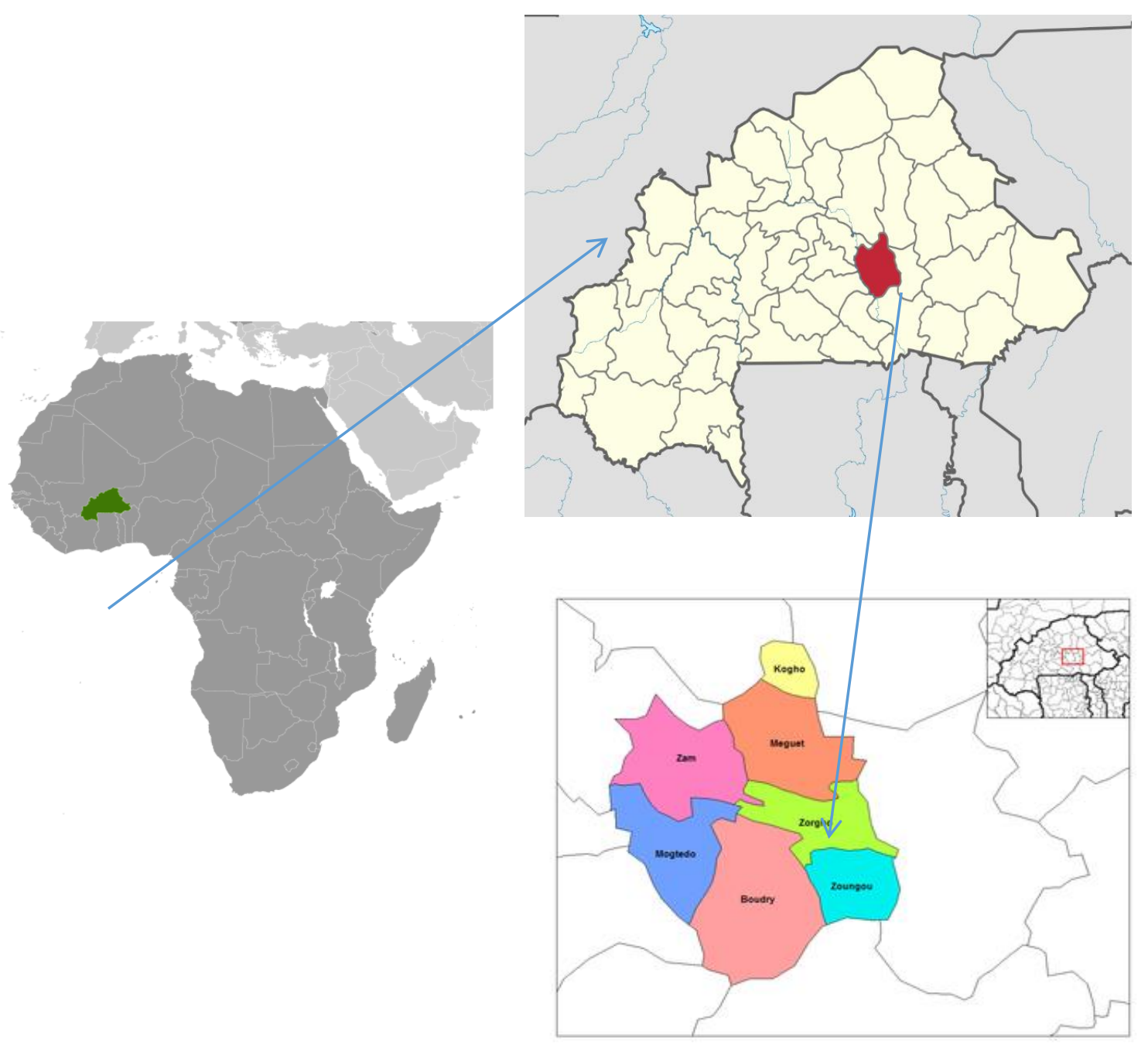

Figure 1: Localisation de la zone concernée par l'étude : province du Ganzourgou avec ses sept communes. 
Tableau 1 : Effectifs et proportion des structures et organisations professionnelles enquêtées.

\begin{tabular}{lcc}
\hline Catégorie d'acteurs & $\begin{array}{c}\text { Nombre de personnes } \\
\text { enquêtées (n) }\end{array}$ & $\begin{array}{c}\text { Proportion } \\
\text { (\%) }\end{array}$ \\
\hline $\begin{array}{l}\text { Services techniques déconcentrés de l'administration } \\
\text { et des collectivités }\end{array}$ & 15 & 12,7 \\
Organisations faîtières de pêcheurs & 27 & 22,7 \\
Organisation des femmes transformatrices des & 16 & 13,4 \\
produits de pêche & 36 & 30,2 \\
Consommateurs & 25 & 21,0 \\
Personnes ressources (notables, anciens pêcheurs) & $\mathbf{1 1 9}$ & $\mathbf{1 0 0}$ \\
Total & & \\
\hline
\end{tabular}

\section{RESULTATS}

Diversité et description des espèces rencontrées dans la province

Deux (02) espèces de grenouilles appartenant à deux familles d'Anoure ont été rencontrées dans les écosystèmes humides de la province du Ganzourgou. Il s'agit par ordre d'importance numérique d'occurrence de :

- Hoplobatrachus occipitalis, Günther 1858, de la famille des Dicroglossidae qui est rencontrée à $95 \%$ dans les captures et paniers de pêche. Elle est bien connue des populations locales sous le nom de «louanga» pour l'ethnie Mossi qui vit dans cette province. Cette grenouille est large et plate avec une palmure complète entre les orteils. Les yeux sont très globuleux et proéminents avec une ligne claire transversale qui relie la partie arrière des deux orbites. La peau du dos forme des plis très courts et discontinus, assez irrégulièrement disposés, ressemblant à des pustules. La coloration dorsale et le dessus des cuisses sont d'un gris brunâtre, plus ou moins sombre, constellé de tâches plus sombres, irrégulières et souvent peu distinctes (Figure 2).

- Xenopus muelleri, Peters 1844, de la famille des Pipidae est connue par la population locale sous le nom de «souansga». C'est une espèce de grosse taille et peut atteindre $72 \mathrm{~mm}$. Les yeux sont relativement gros avec un tentacule sous-oculaire bien développé. La coloration est grise sur le dos, presque blanchâtre sur le ventre et jaune à la partie inférieure des cuisses. La palmure est très développée aux membres postérieurs et les orteils portent trois fortes griffes cornées noires (Figure 3). Elle est peu fréquente $(<5 \%$ d'occurrence) dans les captures en saison sèche.

\section{Habitats et zone d'occurrence des grenouilles}

Les zones d'occurrence, servant $\mathrm{d}$ 'habitat à ces grenouilles sont les écosystèmes humides représentés dans la province d'étude par ordre d'importance décoissante par les lacs de barrage $(40,70 \%)$, suivi par les mares temporaires $(32,20 \%)$, les petites et moyennes retenues d'eaux artificielles $(24,0 \%)$, les cours d'eau intermittentes et les plaines d'inondation pour $2,70 \%$ (Figure 4 ).

\section{Exploitation des grenouilles dans la province}

La collecte des grenouilles dans la province du Ganzourgou vient en appendice de la pêche aux poissons. Aussi, les engins utilisés 
prioritairement pour la capture des poissons servent à l'occasion pour les prises de grenouilles. Ces engins (Figure 5) sont constitués par les filets maillants $(39,45 \%)$, les pièges traditionnels et les nasses $(22,40 \%)$, les hameçons $(21,15 \%)$. Certains de ces engins sont représentés en Figures 6 et 7. Dans des cas erratiques, avec les enfants notamment, les captures manuelles de grenouilles sont opérées dans de petites poches ou collections d'eau et ce mode de capture représenterait environ $17,0 \%$.

L'exploitation des grenouilles est une activité de contre-saison qui s'étale sur la période allant d'octobre à mars/avril, soit environ 5 à 6 mois . Cette période coïncide avec la décrue des eaux et la fin des travaux champêtres de la période d'hivernage. La quasi-totalité des exploitants sont des agropêcheurs qui pratiquent prioritairement la pêche aux poissons et secondairement celle des grenouilles dont la chair est très prisée par les populations autochtones de la province.

\section{Utilisation des grenouilles dans la province}

Les grenouilles connaissent plusieurs types d'utilisation incluant la nutrition humaine et les aspects socio-culturels, y compris la pharmacopée.

La principale utilisation connue des grenouilles dans la province est la consommation humaine. En effet, sur un échantillon de 35 personnes enquêtées, 22 personnes, soit $62,86 \%$, confirment cette destination des grenouilles aux fins de l'alimentation humaine. Le principal mode de transformation des grenouilles pour la consommation est la friture à l'huile. Cette opération met sur le marché local et le long des grands axes routiers des produits frits vendus en tas de trois pièces à 500 FCFA (Figure 8).
Une partie des personnes enquêtées, soit $22,84 \%$, rapportent l'utilisation des grenouilles dans la médecine traditionnelle ou encore la pharmacopée. En effet, les graisses de grenouilles seraient utilisées, selon les enquêtées, pour des soins dermatologiques. Des organes de grenouilles non spécifiés par les enquêtées seraient également utilisés dans le traitement des affections des voies respiratoires (toux, sinusites) ainsi que dans le traitement de la rougeole (application de la chair dès l'apparition des éruptions cutanées).

Sur le plan socio-culturel, les personnes interrogées $(14,30 \%)$ rapportent une utilisation mystique des grenouilles en production agricole pour accélérer la croissance des plantes. Le cœur des grenouilles serait également utilisé dans les préparations mystiques pour une persévérance / une endurance des enfants et des jeunes personnes dans les combats physiques. La peau de ces amphibiens, en raison de son élasticité, est utilisée dans la fabrication du tambour, un instrument de musique local.

\section{Traits de menaces des grenouilles}

Plusieurs facteurs ont été rapportés ou identifiés par les personnes enquêtées comme étant une source de menace pour les populations sauvages de grenouilles dans la province. Ces facteurs sont variés et inclus des origines anthropo-climatiques et écologiques. En effet, sur un effectif de 104 acteurs interrogés, les pollutions agrochimiques (engrais, pesticides, herbicides) et l'utilisation des produits chimiques dans l'exploitation minière sont indexées par 70 personnes, soit $67,30 \%$ de la fréquence de réponses. Les autres facteurs identifiés inclus la pénurie d'eau c'est-à-dire la sécheresse ou tarissement des sources d'eau (24,03\%), la surexploitation $(5,76 \%)$ et la prédation pour $2,91 \%$ (Figure 9 ). 


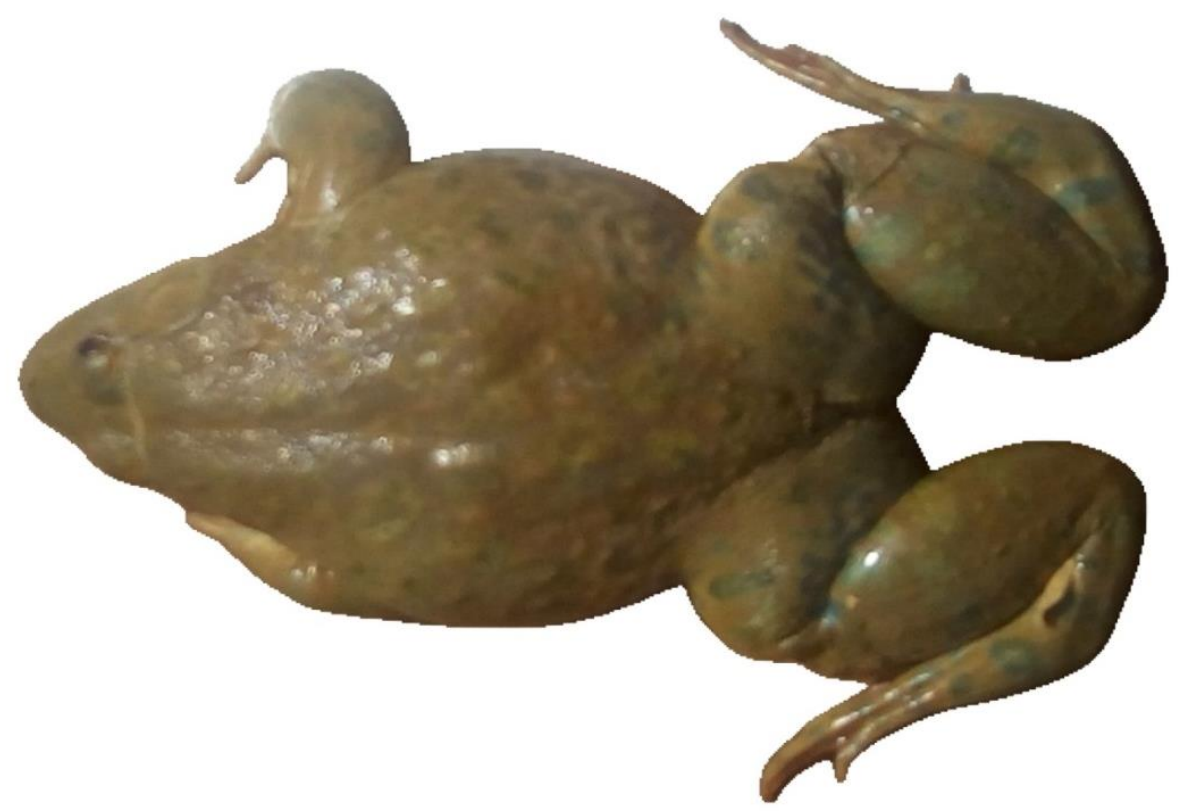

Figure 2 : Hoplobatrachus occipitalis capturée à Mogtédo, province du Ganzourgou (Décembre 2018).

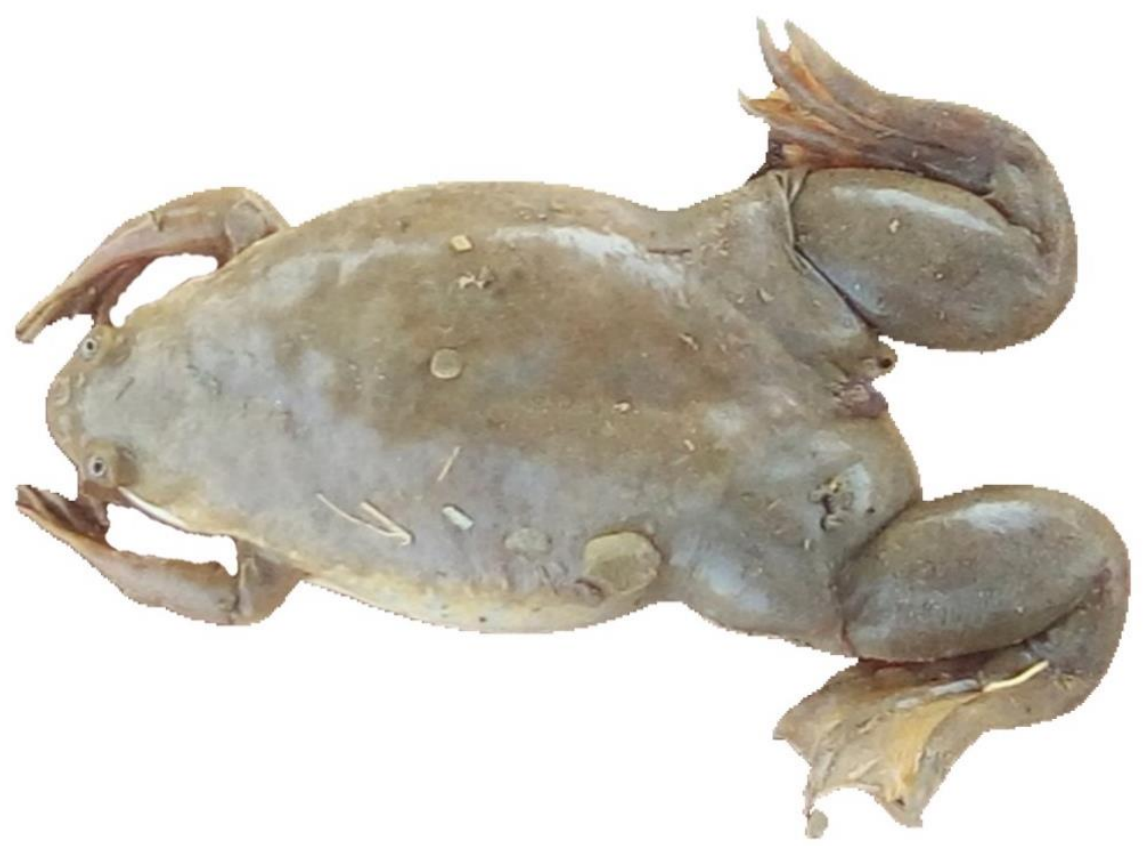

Figure 3 : Xenopus muelleri capturée en saison humide dans un cours d'eau de Sanbrado, province du Ganzourgou (Juillet 2019). 
N. D. COULIBALY et B. ZIGUI / Int. J. Biol. Chem. Sci. 15(3): 1090-1103, 2021

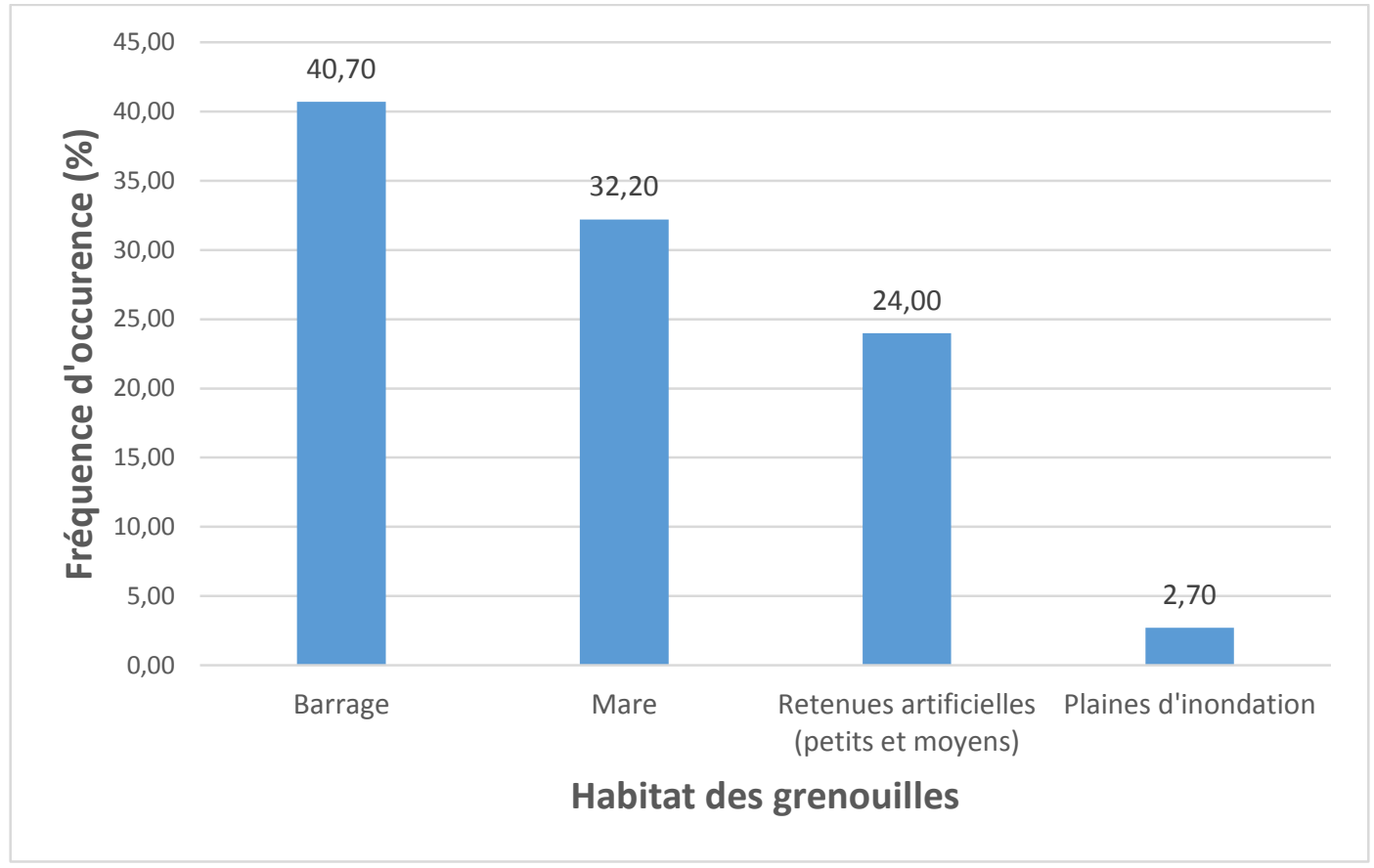

Figure 4 : Habitats des grenouilles dans la province du Ganzourgou.

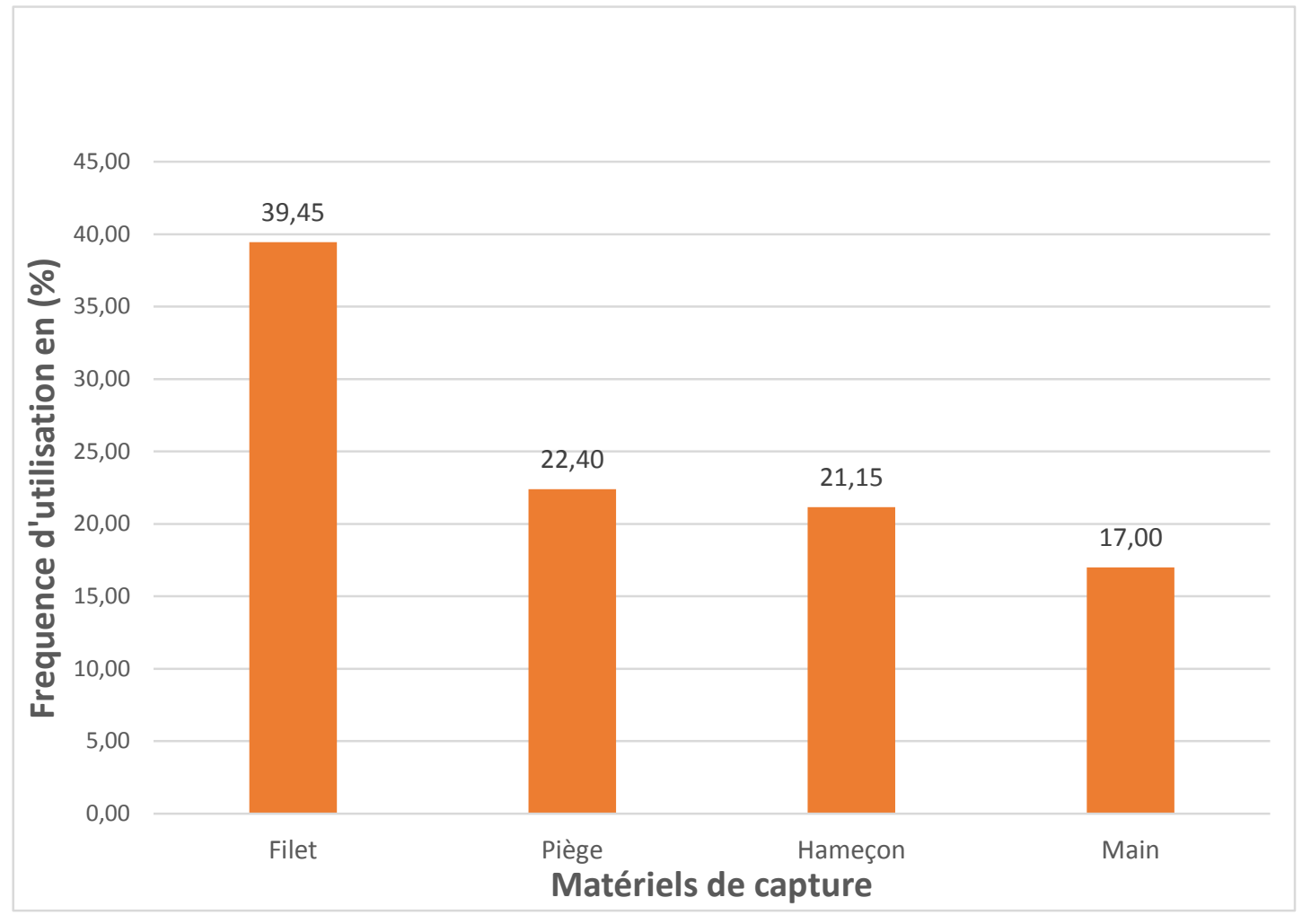

Figure 5 : Engins utilisés pour la capture des grenouilles dans la province du Ganzourgou. 

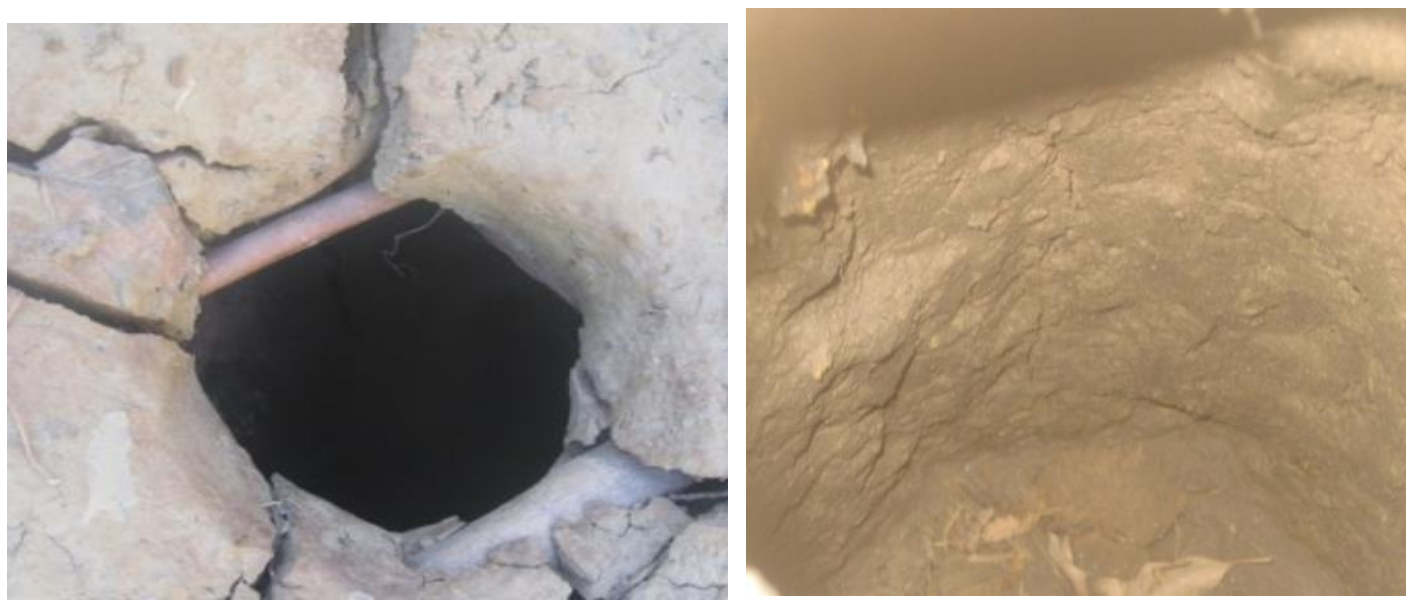

Figure 6 : Piège artisanale pour la capture des grenouilles; vue du piège de l'extérieur (gauche) et la fosse intérieure pour la collecte (droite).

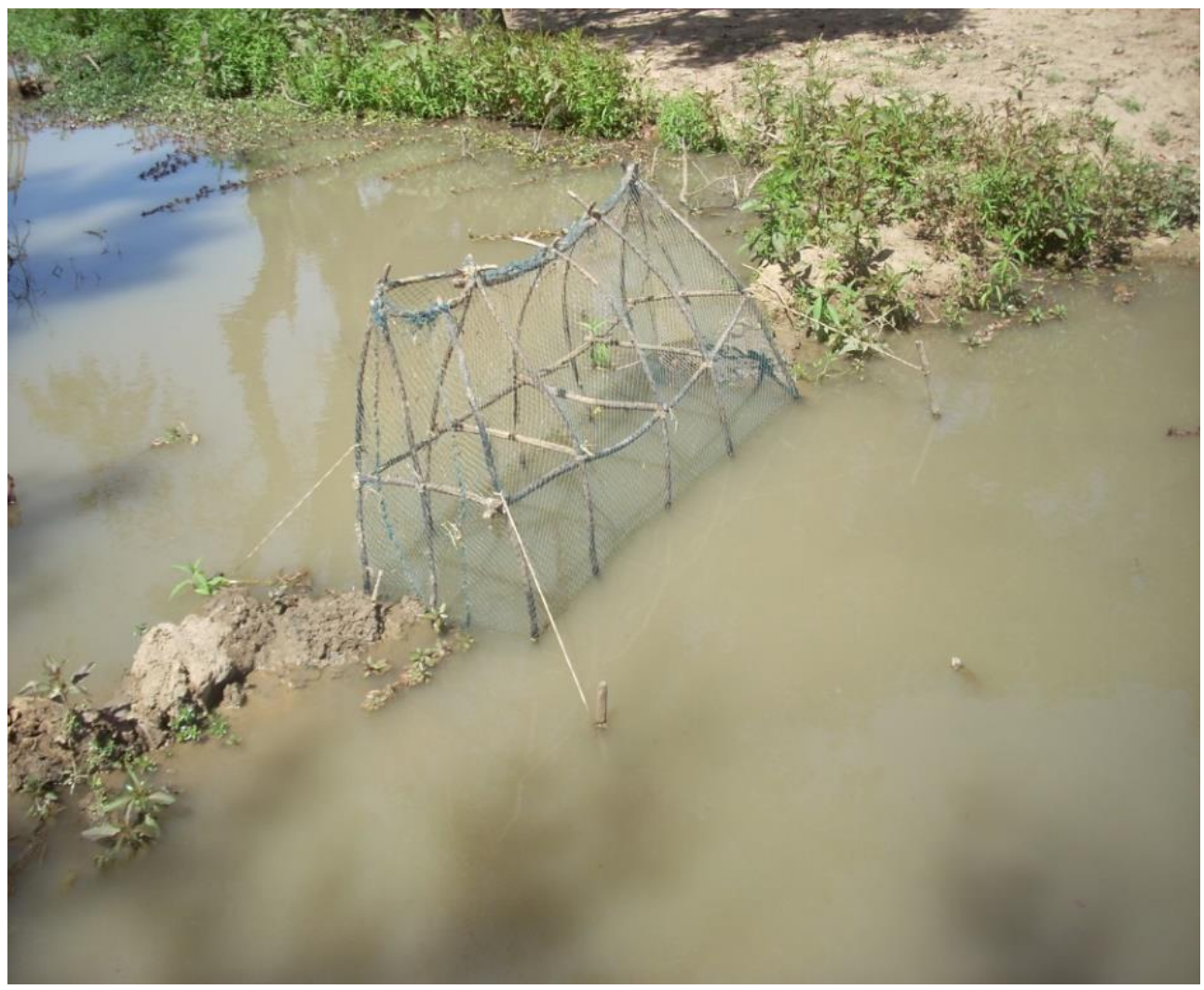

Figure 7 : Grande nasse fixe utilisée pour la capture des poissons benthiques (silures, protoptères, polyptères, ...) et les grenouilles. 


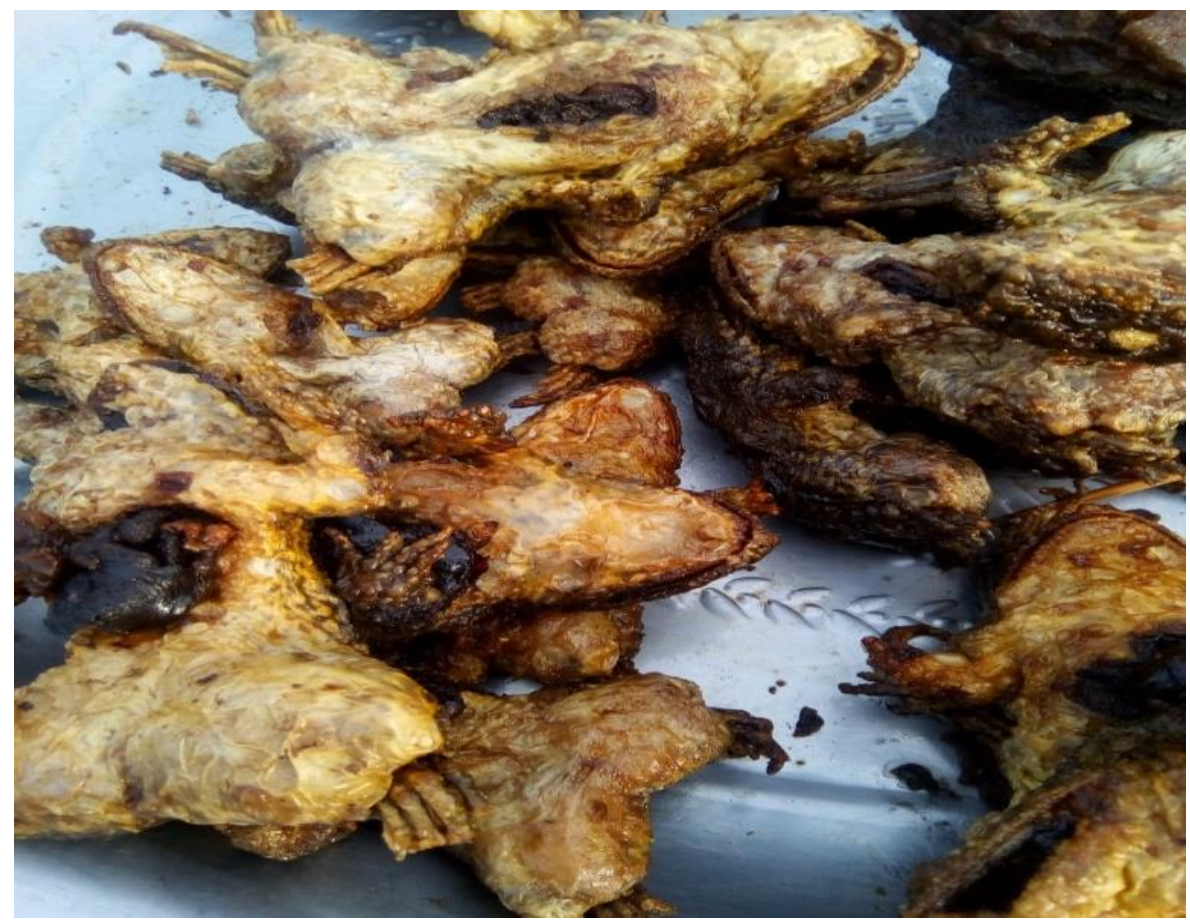

Figure 8 : Grénouilles frites à l'huile et vendues en tas de trois pièces au marché et le long de la route nationale $n^{\circ} 4$.

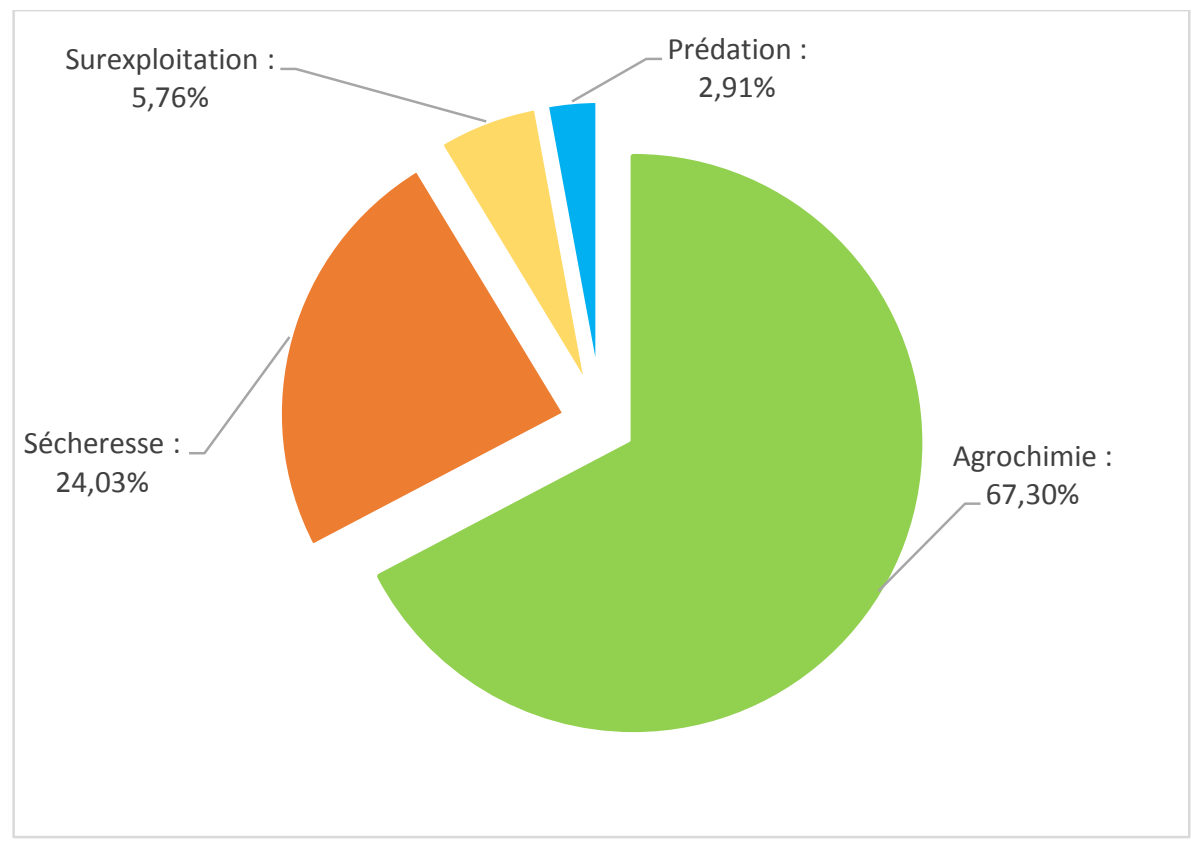

Figure 9 : Importance des facteurs identifiés comme une menace pour les populations sauvages de grenouilles dans la province du Ganzourgou. 


\section{DISCUSSION}

L'exploitation des grenouilles est une activité qui se fait depuis des temps forts anciens pour différents usages dont l'alimentation et la pharmacopée en milieu rural. Sur les quarante et cinq (45) provinces administratives que compte le Burkina Faso, celle du Ganzourgou a la particularité de promouvoir à grande échelle la consommation et le commerce local des grenouilles, voire de certaines espèces de crapauds (Mohneke et al., 2010a). Les espèces rencontrées dans cette province ont été décrites par Mohneke et al., (2010b). Elles font partie des 17 amphibiens (grenouilles et crapauds) enregistrés au Burkina Faso (Mohneke et al., 2010b). Parmi les deux espèces rencontrées dans la province, Hoplobathrachus occipitalis prédomine dans les captures en raison de sa bonne adaptation aux conditions arides de la zone (Penner et al., 2010 ; Kia et al., 2018 ; Oungbe et al., 2018). Marina (2016) rapporte qu'elle serait la seule espèce exploitée dans la province. Quant à Xenopus muelleri, elle a un mode de vie cryptique et peut passer plus de la moitié de l'année enterrée dans la vase, pour ne réapparaitre qu'en début de saison des pluies (Mohneke et al., 2010b ; Penner et al., 2010 ; Onadeko et al., 2011). Elle n'est donc pas fréquente dans les prises de pêche, surtout que la pêche aux grenouilles se pratique plus en saison sèche. Le nombre d'espèces exploitées dans le Ganzourgou est inférieur aux six (6) amphibiens d'intérêt socio-économiques rencontrées au Burkina Faso et qui inclus H.occipitalis, Pyxicephalus edulis, Ptychadena bibroni, $P$. oxyrhynchus, $P$. trinodis et le crapaud Amietophrynus sp (Mohneke et al., 2010c).

L'activité de collecte des grenouilles dans le milieu naturel de la province du Ganzourgou se fait en appendice de la pêche aux poissons par des agro-pêcheurs utilisant une diversité d'engins pour leur capture. La méthode et les engins utilisés sont similaires à ceux rapportés par Mohneke et al. (2010a) et Marina (2016). La chasse nocturne, utilisant des lampes torches pour éblouir les grenouilles est autorisée au Burundi et au Benin (Mohneke et al., 2010c) mais proscrite au Burkina, et particulièrement dans le Ganzourgou où la collecte des grenouilles se fait dans la journée et pendant la période de décrue des eaux. La collecte des grenouilles dans cette province est sous l'emprise de la saison des pluies et de l'hydrologie des cours et plans d'eau. Marina (2016) note deux périodes d'exploitation des grenouilles dans cette province : à la fin de la saison des pluies ou la décrue (septembre à novembre) et de la mi-février à la mi-mai, cette dernière période coïncidant avec les étiages prononcés. Lors de ces étiages, les grenouilles se rassemblent de façon spontanée dans les endroits restés humides ou inondés, ce qui les rend vulnérables aux techniques de capture.

En zone rurale comme dans la province du Ganzourgou, l'exploitation des grenouilles dans le milieu naturel vise d'abord à satisfaire les besoins de couverture en protéines animales au niveau local. La consommation des grenouilles est un trait culturel de l'ethnie « mossi » qui peuple cette province située dans la région du Plateau Central au Burkina Faso. Lors d'une précédente enquête dans cette province, $93 \%$ des personnes interviewées déclarent consommer les grenouilles (Mohneke et al., 2010c). Cette vocation est rencontrée dans la quasi-totalité des pays Africains où les grenouilles sont exploitées. C'est le cas en République Démocratique du Congo, au Burundi, au Cameroun, au Benin, au Nigeria et à Madagascar (Mohneke et al., 2010c ; Onadeko et al., 2011 ; Efenakpo et al., 2016 ; Kia et al., 2018). Si dans le Ganzourgou, l'exploitation des grenouilles vise la satisfaction du marché local, cela n'est pas le cas au Benin, au Nigéria, au Cameroun ou à Madagascar où l'exploitation des grenouilles est orientée vers les marchés extérieurs ou les circuits interurbains pour les grands hôtels (Mohneke et al., 2010c).

A côté de cette première et principale utilisation, les grenouilles sont également utilisées en pharmacopée dans le Ganzourgou. 
Ce rôle a été rapporté par de nombreux auteurs tels Mohneke et al. (2010a, 2010c), Altherr et al. (2011), Onadeko et al. (2011), Efenakpo et al. (2016) et MRAH (2018). Les grenouilles interviennent aussi dans certaines pratiques culturelles «moaga ». Dans certaines sociétés Africaines, les grenouilles sont également utilisées à des fins culturelles (Mohneke et al., 2010c; Efenakpo et al., 2016) et dans la maroquinerie (Onadeko et al., 2011).

Le rôle des grenouilles dans la prévention des maladies à transmission vectorielle comme le paludisme ou le zika est très peu connu des populations du Ganzourgou. Ces grenouilles participent à la destruction (consommation) des larves de ces insectes dans les eaux stagnantes et donc, à la diminution de la prévalence de ces maladies au sein des populations. L'importance des amphibiens dans la destruction des insectes nuisibles en agriculture et en santé publique a été soulignée par certains auteurs (Penner et al., 2010; Altherr et al., 2011; Oungbe et al., 2018). Mohneke et al., (2010a) ont indiqué que le déclin des populations de grenouilles avait entrainé une augmentation des insectes dans les pépinières de riz. Cette situation a obligé les paysans à faire recours à des insecticides chers et insalubres pour éliminer ces insectes. La destruction des insectes dans l'environnement est l'un des rôles autrefois attribués aux grenouilles.

Dans la province du Ganzourgou, les pollutions agrochimiques et l'utilisation des substances chimiques dans l'exploitation minière artisanale sont indexées comme les facteurs de menace les plus importants pour les stocks naturels de grenouilles. Une enquête conduite une décennie auparavant, avait montré que la pénurie d'eau (sécheresse, assèchement des points d'eau) était la première cause de menace ( $46 \%$ des réponses) pour les grenouilles au Burkina Faso (Mohneke et al., 2010c). Selon les pêcheurs lors de la même enquête, la dégradation de l'habitat des grenouilles serait la première et principale cause de déclin (32\% des réponses) suivie par la surexploitation et la pénurie d'eau ( $23 \%$ des réponses). La destruction et la modification de l'habitat, l'exploitation abusive, les pollutions, le réchauffement climatique et les maladies, dont la chytridiomycose en particulier sont également perçus comme les causes de déclins des populations de grenouilles (Mohneke et al., 2010b ; Penner et al., 2010 ; Onadeko et al., 2011). L'un des facteurs de menace le plus préjudiciable aux ressources naturelles «négligées » comme les grenouilles au Burkina Faso est le vide juridique qui entoure leur exploitation. Dans la quasi-totalité des pays Africains, il n'existe aucun mécanisme de régulation/règlementation de l'exploitation des grenouilles en dépit des signes de déclin observés tant au niveau local, national ou régional (Altherr et al., 2011). Au Burkina Faso et dans la province du Ganzourgou en particulier, l'exploitation des grenouilles est une activité non encadrée et non règlementée. Le vide juridique relatif à l'exploitation et la conservation de cette ressource bio-aquatique est la menace la plus importante pour leur sauvegarde.

\section{Conclusion}

Les grenouilles constituent une ressource «négligée ou oubliée » dont l'exploitation rationnelle contribue à la couverture des besoins en protéines animales, surtout dans les zones rurales défavorisées. Les populations natives de la province du Ganzourgou au Burkina Faso, ont fait de la consommation des amphibiens (crapauds et grenouilles) un trait culturel de leur alimentation. Les deux espèces de grenouilles rencontrées dans la province sont exploitées traditionnellement sans aucune mesure de préservation. A côté de l'importante utilisation des substances chimiques et agrochimiques, l'absence de dispositifs règlementaires constitue l'un des facteurs de menace le plus préjudiciable à la préservation de cette ressource bio-aquatique. Les populations sauvages de grenouilles comestibles risquent fort de disparaitre du fait de la surexploitation 
et de l'intensification des productions végétales ou de l'usage des substances chimiques dans l'exploitation minière. Dans un tel contexte, il convient d'entrevoir dès à présent les stratégies de domestication et d'élevage de ces espèces et encourager les efforts de leur conservation.

\section{CONFLIT D'INTERETS}

Les auteurs déclarent qu'en ce qui concerne le présent article, il n'y a aucun conflit d'intérêt.

\section{CONTRIBUTIONS DES AUTEURS}

Chaque auteur a participé à la définition de la thématique, puis à la collecte des données sur le terrain à travers les séances d'animation des focus groups, des interviews, les visites de sites de capture et des marchés locaux. L'élaboration du premier draft de ce document a été participative. La version finale du manuscrit a été lue et approuvée par chacun des auteurs avant son envoi à la revue.

\section{REMERCIEMENTS}

Nous remercions Mme YEYE/GO Jeanne d'Arc, Directrice Provinciale des Ressources Animales et Halieutiques de la province du Ganzourgou pour son assistance et sa franche collaboration lors des sorties sur le terrain. Nous remercions également messieurs COMPAORE René et SAWADOGO Alain de la ZATE de Mogtédo, monsieur ZOUGMORE Mahamadi et monsieur SAWADOGO Zounongo respectivement de la ZATE de Boudry et de Zoungou qui ont facilité les contacts avec les acteurs de leur zone d'intervention. Nos remerciements s'adressent également aux associations de pêcheurs et de femmes transformatrices de grenouilles pour leur aimable collaboration lors de la collecte des données.

\section{RÉFÉRENCES}

Altherr S, Goyenechea A, Schubert D. 2011. Canapes to Extinction: The International Trade in Frogs' Legs and its Ecological Impact. Pro Wildlife, Defenders of
Wildlife and Animal Welfare Institute (eds.): Munich (Germany)-Washington D.C. (USA).

Dipama JM. 2010. L'hydrologie. In Atlas de la Biodiversité de l'Afrique de l'Ouest,: Burkina Faso (Tome II), Thiombiano A, Kampmann D (eds). Bundesministerium für Bildung und Forschung: Frankfurt/Main; 134-137.

Efenakpo OD, Ayodele IA, Ijeomah HM. 2016. Assessment of frog meat utilisation in Ibadan, Oyo State, Nigeria. Journal of Research in Forestry, Wildlife \& Environment, 8(3): 31-43.

Godome T, Tossavi E, Ouattara NI, Fiogbe ED. 2018. Bibliographic synthesis on biology and ecology of Hoplobathrachus occipitalis (Günther, 1858). Int. J. Biol. Chem. Sci., 12(3): 1484-1493. DOI: https://dx.doi.org/10.4314/ijbcs.v12i3.33

Kia GSN, Tijjiani FY, Otolorin RG. 2018. An evaluation of intestinal parasite in edible frogs (Hoplobatrachus sp) sold for consumption in Zaria, Kaduna State, Nigeria. Nigerian Veterinary Journal, 39(3): 209-216. DOI: https://dx.doi.org/10.4314/nvj.v39i3.4

Marina N. 2016. Evaluation du potentiel des ressources halieutiques non ichtyennes: cas des grenouilles dans la province du Ganzourgou. Mémoire d'Inspecteur des Eaux \& Forêts, ENEF de Dinderesso, Bobo-Dioulasso, $90 \mathrm{p}$.

MINEFID (Ministère de l'Economie, des Finances et du Développement). 2015. Monographie de la province du Ganzourgou (Burkina Faso). Direction Régionale de l'Economie et de la Planification (DREP) du Plateau Central (PLC), DREP/PLC, Ziniaré, Burkina Faso.

MRAH (Ministère des Ressources Animales et Halieutiques). 2018. Etat des ressources halieutiques non ichtyennes au Burkina Faso. Rapport d'étude, Direction Générale des Ressources Halieutique : Ouagadougou-Burkina Faso. 
Mohneke M, Hirschfeld M, Rödel MO. 2010a. Utilisation non durable des grenouilles en Afrique de l'Ouest. In Atlas de la Biodiversité de l'Afrique de l'Ouest: Burkina Faso (Tome II), Thiombiano A, Kampmann D (eds). Bundesministerium für Bildung und Forschung: Frankfurt/Main; 108-109.

Mohneke M, Zongo B, Rödel MO. 2010b. Les amphibiens. In Atlas de la Biodiversité de l'Afrique de l'Ouest: Burkina Faso (Tome II), Thiombiano A, Kampmann D (eds). Bundesministerium für Bildung und Forschung: Frankfurt/Main; 298-302.

Mohneke M, Hirschfeld M, Rödel MO, Onadeko AB. 2010c. Dried or fried; amphibians in local and regional food markets in West Africa. Traffic Bulletin, 22(3): 117-128.

Onadeko AB, Egonmwan RI, Saliu JK. 2011. Edible amphibian species: local knowledge of their consomption in South West Nigeria and their nutritional value. West African Journal of Applied Ecology, 19: 67-76.

Oungbe KV, Adeba PJ, Blahoua KG, N'Douba V. 2018. Systematic inventory of anuran species (amphibians) in three agroindustrial zones in the South East of Cote
d'Ivoire. Journal of Applied Biosciences, 131: 13271-13283. DOI: https://dx.doi.org/10.4314/jab.V131 i1.3

Penner J, Adeba PJ, Hillers A, Nago SGA, Rödel MO. 2010. Amphibiens de l'Afrique de l'Ouest. In Atlas de la Biodiversité de l'Afrique de l'Ouest: Burkina Faso (Tome II), Thiombiano A, Kampmann D (eds). Bundesministerium für Bildung und Forschung: Frankfurt/Main; 102-107.

Teixeira RD, Pereira-Mello SCR, Lima dos Santos CAH. 2001. The world market of frog legs. FAO/Globefish Research Programme, Rome.

West African Resources (WAR), Société Minière de Sanbrado (SOMISA). 2019. Etat initial de l'environnement biologique : faune, flore et vie aquatique. BEGE-Rapport d'étude, Ouagadougou- Burkina Faso.

Zigui B. 2020. Grenouilles d'intérêt socioéconomique dans la commune rurale de Mogtédo, Province du Ganzourgou: exploitation et traits de menace. Mémoire de Technicien Supérieur d'Elevage, ENESA/MRAH, Ouagadougou-Burkina Faso, $54 \mathrm{p}$. 